\title{
Dense Matter Characterization by X-ray Thomson Scattering
}

O. L. Landen, S. H. Glenzer, M. J. Edwards, R. W. Lee, G. W. Collins, R. C. Cauble, W. W. Hsing, and B. A. Hammel

This article was submitted to Radiative Properties of Hot Dense Matter Santa Barbara, California October 30 - November 2, 2000

\section{December 29, 2000}




\section{DISCLAIMER}

This document was prepared as an account of work sponsored by an agency of the United States Government. Neither the United States Government nor the University of California nor any of their employees, makes any warranty, express or implied, or assumes any legal liability or responsibility for the accuracy, completeness, or usefulness of any information, apparatus, product, or process disclosed, or represents that its use would not infringe privately owned rights. Reference herein to any specific commercial product, process, or service by trade name, trademark, manufacturer, or otherwise, does not necessarily constitute or imply its endorsement, recommendation, or favoring by the United States Government or the University of California. The views and opinions of authors expressed herein do not necessarily state or reflect those of the United States Government or the University of California, and shall not be used for advertising or product endorsement purposes.

This is a preprint of a paper intended for publication in a journal or proceedings. Since changes may be made before publication, this preprint is made available with the understanding that it will not be cited or reproduced without the permission of the author.

This report has been reproduced directly from the best available copy.

Available electronically at http://www.doc.gov/bridge

Available for a processing fee to U.S. Department of Energy

And its contractors in paper from

U.S. Department of Energy

Office of Scientific and Technical Information

P.O. Box 62

Oak Ridge, TN 37831-0062

Telephone: (865) 576-8401

Facsimile: (865) 576-5728

E-mail: reports@adonis.osti.gov

Available for the sale to the public from

U.S. Department of Commerce

National Technical Information Service

5285 Port Royal Road

Springfield, VA 22161

Telephone: (800) 553-6847

Facsimile: (703) 605-6900

E-mail: orders@ntis.fedworld.gov

Online ordering: http:/ / www.ntis.gov/ordering.htm

\section{OR}

Lawrence Livermore National Laboratory

Technical Information Department's Digital Library

http: / / www.llnl.gov/tid/Library.html 


\title{
Dense Matter Characterization by X-ray Thomson Scattering*
}

\author{
O. L. Landen, S.H. Glenzer, M.J. Edwards, R.W. Lee, G.W. Collins, R.C. Cauble, W.W. Hsing \\ and B.A. Hammel
}

Lawrence Livermore National Laboratory

P.O. Box 5508, Livermore, CA 94551

\begin{abstract}
We discuss the extension of the powerful technique of Thomson scattering to the $\mathrm{x}$-ray regime for providing an independent measure of plasma parameters for dense plasmas. By spectrallyresolving the scattering, the coherent (Rayleigh) unshifted scattering component can be separated from the incoherent Thomson component, which is both Compton and Doppler shifted. The free electron density and temperature can then be inferred from the spectral shape of the high frequency Thomson scattering component. In addition, as the plasma temperature is dcreased, the electron velocity distribution as measured by incoherent Thomson scattering will make a transition from the traditional Gaussian Boltzmann distribution to a density-dependent parabolic Fermi distribution to. We also present a discussion for a proof-of-principle experiment appropriate for a high energy laser facility.
\end{abstract}

Keywords: dense plasmas, strongly coupled plasmas, x-rays, Thomson scattering, Compton scattering

\footnotetext{
*Work performed under the auspices of the U.S. Department of Energy by the University of California Lawrence Livermore National Laboratory under contract number W-7405-ENG-48.
} 


\section{Introduction}

The microscopic behavior of solid density plasmas has been the subject of numerous theoretical studies [1-4] but no known definitive experiments. There has been a recent surge in interest in understanding solid density plasma properties, partially motivated by the emergence of shortpulse high power lasers that have the ability to heat solids before significant hydrodynamic motion occurs.[5,6]. Currently, microscopic experimental techniques are limited, as optical probing only provides information on rarefied surface layers since solid density plasmas are opaque to visible and UV light. The matter of interest is at sufficiently low temperature that emission spectroscopy is not possible. Absorption spectroscopy can only be employed at higher energy as the transmission of probe sources will be too small for outer shell line transitions.

To make progress in this regime, we propose a powerful, alternative diagnosis method, spectrally-resolved multi-keV x-ray scattering. By spectrally discriminating between the coherent (Rayleigh), Compton and Thomson scattering components, we expect to gather information on several microscopic parameters, including the free and bound electron densities and fractions, temperature, plasma flow velocity, and plasma collisionality. We will further show that the Omega facility at the Laboratory for Laser Energetics can provide the required photon flux for single-shot detection and the required uniform heating by virtue of its many beams covering a full sphere.

The method will be to prepare a uniformly heated (up to $10 \mathrm{eV}$ ) solid density mm-scale Be plasma by volumetric heating using multi-keV x-rays from laser created solid target plasmas. Another multi-keV line radiator, at wavelength $\lambda$, produced from a second delayed laser plasma will provide the narrow $(\Delta \lambda / \lambda<.003)$ line required for $\mathrm{x}$-ray Thomson scattering before the Be plasma cools or disassembles. The near back-scattered photons will be collected and spectrally 
dispersed by a high reflectance Bragg crystal coupled to a gated framing camera. We expect to be able to determine both the Fermi energy $\mathrm{T}_{\mathrm{F}}$ (and hence free electron density) and electron temperature from the high frequency component of the Thomson scattered spectrum. We expect this first attempt at extending the versatility of laser Thomson scattering [1] to the x-ray regime to lead to detailed dense plasma studies of great interest to the high energy density and plasma physics communities.

\section{Motivation}

The full range of plasmas, from Fermi degenerate, to strongly coupled, to high temperature ideal gas plasmas are present at high density in a variety of laboratory [7-13] and astrophysical environments.[14-16] The Fermi degenerate plasmas can be characterized by $T_{e}<T_{F}$, the strongly coupled plasmas by a combination of $\mathrm{T}_{\mathrm{e}}>\mathrm{T}_{\mathrm{F}}$ and the ratio $\Gamma_{\text {ee }}$ of the Coulomb potential energy between free electrons to the kinetic energy of the free electrons being $>1$, and the ideal

plasmas by $\Gamma_{\mathrm{ee}}<1$. These are states of matter occurring at some location during the interaction between intense lasers and a solid. These are also regimes accessed by the DT fuel during an ICF implosion.

In Figure 1, the strongly coupled plasma $\left(\Gamma_{\mathrm{ee}}>1\right.$ and $\left.\mathrm{T}_{\mathrm{e}}>\mathrm{T}_{\mathrm{F}}\right)$ and Fermi degenerate regimes $\left(\mathrm{T}_{\mathrm{e}}\right.$ $<\mathrm{T}_{\mathrm{F}}$ ) are shown shaded in electron density $\mathrm{n}_{\mathrm{e}}$ - electron temperature $\mathrm{T}_{\mathrm{e}}$ space. For a given density at the lowest temperatures, the plasmas are either Fermi degenerate or only partially ionized, and hence in a sense only weakly coupled. At higher temperatures, they behave as ideal gases with insignificant inter-particle coupling. In between, the ideal gas approximation for plasma behavior breaks down. The concept of a Debye screening length breaks down since the Debye length $\lambda_{\mathrm{D}}$ becomes less than the average interparticle spacing. Various statistical mechanics models [18-20] differ by factors of several in the predicted electron-ion collisionality 
in this regime. Material properties such as electrical [21-24] and thermal conductivity $[25,26]$, opacity [27-30], and equation-of-state (EOS) [31,32] have been studied in this regime to attempt to resolve theoretical and calculational uncertainties. However, the usefulness of such measurements has been impaired because of the lack of an independent measurement of temperature and density.

Moreover, the optical experiments conducted so far have either probed low density plasmas amenable to internal optical probing $[27,33,34]$, or attempted to infer conditions by probing in reflection mode [35]. Figure 1 indicates that to probe at depth the low density, strongly coupled regime with an optical probe, one must work at $\mathrm{eV}$ or sub-eV temperatures. This leads to either partial ionization and hence the complication of neighboring bound states and dominance of electron atom collisions, or the production of a transient over-ionized non-equilibrium state which will quickly recombine by three-body recombination. Surface probing of any overdense plasmas is difficult to interpret [6,35-39] because density gradient scalelengths of the order of $\lambda / 2 \pi$ dramatically modify observables such as reflectivity and phase modulation $[36,40,41]$. Internal x-ray probing for plasmas at densities near solid and above has relied, so far, on continuum edge spectroscopy and extended x-ray absorption fine-structure (EXAFS) [42-46], line shape spectroscopy [10,47], or non-spectrally resolved x-ray scattering [48-51]. However, the interpretation of results from all such techniques rely on knowledge of the ionization balance, density and temperature.

We discuss here an extension of spectrally resolved Thomson scattering to the x-ray regime, for direct measurements of the ionization state, density, temperature, and the microscopic behavior of dense plasmas. This would be the first direct measurement of microscopic parameters of solid density plasmas, which eventually could be used to properly interpret laboratory measurements of material properties such as thermal and electrical conductivity, EOS and opacity. 


\section{X-ray Thomson Scattering}

Thomson scattering [52-55] at a probe wavelength $\lambda$ and angle $\theta$ is characterized by the scattering parameter $\alpha$, proportional to the ratio of the probing scale-length $\lambda_{s}=\lambda / 2 \sin (\theta / 2)$ to the Debye length:

$\alpha=\lambda_{\mathrm{s}} / 2 \pi \lambda_{\mathrm{D}}$

For $\alpha<1$, spectrally-resolved incoherent Thomson scattering provides information on the electron velocity distribution function $f(v)$ and directed velocity of free electrons from the Doppler shifts experienced by scattered probe photons. For $\alpha>1$, the collective scattering regime, the scattering is sensitive to temporal correlations between electron motion separated by more than a Debye length and hence the scattering is dominated by ion-acoustic and electron plasma wave resonances, the latter set by the Bohm-Gross dispersion relation. The frequency shift of the resonance is dependent on density through the plasma frequency, while the width of the resonances yields information on wave damping. In the intermediate regime near $\alpha=1$, the form of the electron plasma high frequency component depends strongly on both the electron temperature and density, providing a robust internal measurement of these basic plasma parameters, as confirmed by spectroscopy [56].

Plotted on Fig. 1 are the regimes accessible by Thomson scattering with $\alpha=0.1-0.3$ and $\theta=180^{\circ}$ for a UV and an X-ray probe wavelength $\lambda$. By Eq. (1), such Thomson scattering accesses regimes in which the Debye length is of order the probe wavelength (e.g., $\lambda_{D} \approx 1000 \AA$ for $a$ $2400 \AA$ probe). By switching from a UV probe at $2400 \AA$ to an $\mathrm{x}$-ray probe at $2.4 \AA$, we can effectively probe solid density plasmas with Debye lengths of the order of the interparticle 
spacing or shorter. Stated differently, for a given plasma temperature, we should be able to access a density that is six orders of magnitude higher than previously attempted. In particular, Fig. 1 shows that the solid density regime (shown for beryllium) is accessible to $2.4 \AA$ Thomson scattering.

It is interesting to consider the $T_{e}-n_{e}$ parameter space covered at a constant value of the scattering parameter $\alpha$ as one transitions from the ideal to Fermi degenerate plasma regime. In the case of ideal plasmas, $\alpha \sim 1 / \lambda_{D} \sim \sqrt{ }\left(T_{e} / n_{e}\right)$, so constant $\alpha$ corresponds to a line of slope +1 on Fig. 1. For Fermi degenerate plasmas, the Debye length is no longer determined simply by the ionized "free" electron density. Only those electrons at the top of the Fermi surface can participate in momentum changing collisions and hence in shielding, with the fraction $=(3 / 2)\left(T_{e} / T_{F}\right)$. Including this fractional term in the formula for $\lambda_{D}$ and hence $\alpha$ leads to:

$\alpha \sim \sqrt{ }\left\{(3 / 2) \mathrm{n}_{\mathrm{e}}\left(\mathrm{T}_{\mathrm{e}} / \mathrm{T}_{\mathrm{F}}\right) / \mathrm{T}_{\mathrm{e}}\right\} \sim \sqrt{ }\left(\mathrm{n}_{\mathrm{e}} / \mathrm{T}_{\mathrm{F}}\right) \sim \mathrm{n}_{\mathrm{e}}{ }^{1 / 6}$

Hence, for Fermi degenerate plasmas, one finds [57] that the scattering parameter $\alpha$ is independent of $\mathrm{T}_{e}$, as shown by the horizontal low temperature part of the $\alpha=0.3, \lambda=2.4 \AA$ line on Fig. 1. Accessing the collective scattering regime $\alpha>1$ will require either smaller scattering angles, considerably higher densities and/or longer wavelength probes.

For spectrally resolved x-ray Thomson scattering, one does not necessarily need an x-ray laser [58] for the following reasons. First, information on solid density plasmas can be obtained by resolving the high frequency Thomson scattered components, so that only probe line widths $\Delta \lambda / \lambda$ of order $\mathrm{v} / \mathrm{c}$ are required, $=.003$ at a few $\mathrm{eV}$ electron temperature. These can be provided by resonance lines from hot mid $\mathrm{Z}$ plasmas. Second, Thomson scattering from individual electrons $(\alpha<1$ regime) is incoherent and hence will require little or no probe transverse coherence. 
Third, the fraction of scattered photons will be substantial. For example, for $n_{e}=1.6 \times 10^{23} \mathrm{~cm}^{-3}$, the Thomson scattering cross-section of $\sigma_{\mathrm{T}}=6.6 \times 10^{-25} \mathrm{~cm}^{-2}$, and a path length $\mathrm{x}$ of $0.1 \mathrm{~cm}$ accessible in low $Z$ material, the fraction scattered $=n_{e} \sigma x=0.01$, close to the maximum desirable for avoiding multiple scatterings. Coupled with a realistic source solid angle of 0.1 steradians, the scattered fraction is $10^{-4}$, which is substantially larger than that available for visible Thomson scattering at lower densities.

A schematic of the expected generic backscattered spectrum is shown in Fig. 2. Coherent scattering [59] from tightly bound electrons $\left(Z_{t b}\right.$ per atom) should provide an unshifted peak at the probe wavelength whose intensity varies as $Z_{\mathrm{tb}}{ }^{2}$. Incoherent Compton scattering from weakly bound (binding energy $<2(\mathrm{~h} v)^{2} / \mathrm{m}_{\mathrm{e}} \mathrm{c}^{2}$ ) electrons [60-62] ( $\mathrm{Z}_{\mathrm{wb}}$ per atom) should provide a second peak downshifted in energy by $2 \mathrm{hv} / \mathrm{mc}^{2}$, with an intensity varying as $Z_{\mathrm{wb}}$. Compton and Thomson scattering from free electrons $\left(Z_{\mathrm{f}}\right.$ per atom) should provide a dispersed spectrum centered on the Compton peak, with a spectrally integrated intensity varying as $Z_{\mathrm{f}}$. The form of the Thomson scattered spectrum will in general depend on the Fermi energy $T_{F}$, free electron density $n_{e}$, temperature $T_{e}$, electron-ion collisionality $v_{e i}$, and strong coupling parameter $\Gamma$. In the limit $\mathrm{T}_{\mathrm{e}} / \mathrm{T}_{\mathrm{F}}<1$ and $\alpha<<1$, the incoherent scattered distribution function from electrons will be dependent on $T_{e}$ and $T_{F}$ and is given by $\mathrm{f}(\Delta \mathrm{v} / \mathrm{v})=\mathrm{f}\left\{2\left(\mathrm{v}_{\mathrm{x}} / \mathrm{v}_{\mathrm{F}}\right)\left(\mathrm{v}_{\mathrm{F}} / \mathrm{c}\right) \sin (\theta / 2)\right\}$, where $\mathrm{f}\left(\mathrm{v}_{\mathrm{x}} / \mathrm{v}_{\mathrm{F}}\right)$ is given by:

$$
f\left(\frac{v_{x}}{v_{F}}\right) d\left(\frac{v_{x}}{v_{F}}\right) \propto \int_{0}^{\pi / 2} \frac{\left(\frac{v_{x}}{v_{F} \cos \beta}\right)^{2} \tan \beta d \beta}{\exp \left[\left(\left(\frac{v_{x}}{v_{F} \cos \beta}\right)^{2}-1+\left(\frac{\pi^{2}}{12}\right)\left(\frac{T_{e}}{T_{F}}\right)^{2}\right) /\left(\frac{T_{e}}{T_{F}}\right)\right]+1} d\left(\frac{v_{x}}{v_{F}}\right)
$$


and where $\mathrm{v}_{\mathrm{x}}$ is the component of the electron velocity in the $\overline{\mathbf{x}}=\overline{\mathbf{k}}_{\mathrm{s}}=\overline{\mathbf{k}}_{\text {scat }}-\overline{\mathbf{k}}_{\mathrm{I} / \mathbf{P}}$ direction, $\mathrm{v}_{\mathrm{F}}$ is the Fermi velocity $=\sqrt{ }\left(2 \mathrm{kT}_{\mathrm{F}} / \mathrm{m}_{\mathrm{e}}\right), \beta$ is the angle between the electron velocity direction and the $\mathrm{x}$ axis, and $\Delta v$ is the frequency shift from the Compton shifted position. The term $\left(\pi^{2} / 12\right)\left(\mathrm{T}_{\mathrm{e}} / \mathrm{T}_{\mathrm{F}}\right)^{2}$ accounts for the fact that the chemical potential $\mu$ in the expression for the occupation of states for fermions, $1 /\{\exp [(\mathrm{E}-\mu) / \mathrm{Te}]+1\}$, has some temperature dependence at finite temperature [63].

Examples of calculated Thomson scattered spectra using Eq. (3) are shown on Fig. 3 as a function of various values of $\mathrm{T}_{\mathrm{e}} / \mathrm{T}_{\mathrm{F}}$ (for $\mathrm{T}_{\mathrm{F}}=15 \mathrm{eV}, \theta=180^{\circ}$ ). In the limit of $\mathrm{T}_{\mathrm{e}}=0$, the form of the scattered distribution function is parabolic [64], making a transition to the familiar Gaussian distribution in the case of Boltzmann statistics $\left(\mathrm{T}_{\mathrm{e}}>>\mathrm{T}_{\mathrm{F}}\right)$. Clearly measurements on the tail of the distribution are most sensitive to the ratio $\mathrm{T}_{\mathrm{e}} / \mathrm{T}_{\mathrm{F}}$.

Hence, by spectrally resolving the scattered x-ray spectrum, we would gain access, for the first time, to an unparalleled source of information on warm-to-hot dense matter. For example, we should be able to infer $Z_{f}, Z_{t b}$, and $Z_{w b}$.from the relative intensities of coherent, incoherent and free electron scattering contributions. This would allow us to discriminate between different ionization balance models [51] such as Thomas-Fermi and Inferno [65]. We should be able to infer the free electron temperature, Fermi energy $\mathrm{T}_{\mathrm{F}}$, hence electron density (and ionization state in cases where the ion density is known or effectively hydrodynamically frozen) from the shape of the Thomson scattered spectrum for $\alpha<1$. Furthermore, for strongly coupled plasmas, one of the more intriguing possibilities is the establishment of long-range coherence even in the plasma state. In that case, one would need to increase the transverse coherence length of our incoherent source, easily accomplished by further distancing the source from the sample. If coherent plasma wave modes exist, then the Thomson scattering contribution should increase as the square of the number of coherent scatterers. 


\section{Experimental Design}

We have designed an x-ray scattering experiment optimized for the Omega laser facility [66] capabilities which should provide high quality spectral data on a single shot. A detailed view of the target design is shown in Fig. 4. The emphasis here is to create a uniformly heated largescale sample with known energy content that can be probed internally before it cools or disassembles appreciably. The previous experiments have either been performed in the presence of unknown density and temperature gradients, or unknown temperatures, requiring hydrodynamic modeling to constrain the interpretation of the data. The percentage of the sample subject to shock compression and disassembly by rarefaction after preheating is minimized by minimizing the surface area to volume of the sample. The symmetric arrangement of beams permits the use of a cylindrical sample overcoated on its curved surface with a thin high Z x-ray conversion layer transparent to its own multi-keV x-rays for providing the pump source (see Fig. 4). 42-45 of the 60 laser beams can be focussed onto this curved surface at incidence angles below $45^{\circ} \cdot 10-12$ of the remaining beams can be used to provide a bright $\mathrm{x}$-ray probe source using a mid Z x-ray conversion foil in the shape of an annulus. The complete target is cylindrically symmetric, making it amenable to direct two-dimensional (2D) modeling.

The uniformity of heating is optimized here by using multi-keV x-rays over a narrow band (4-5 $\mathrm{keV}$ ) to provide volumetric heating, and by enveloping the sample by the pump source distributed over approximately $3 \pi$ steradians (see Fig. 5a). The pump photon mean free path has been chosen to be twice the sample diameter to provide acceptable uniformity in the energy absorbed / sample atom at all positions inside the sample. Fig. 5b shows the results of viewfactor calculations, plotting the distribution of energy absorbed per atom throughout the central volume of the Be cylinder. By adding an overhang section, as shown on Fig. 5a, we can 
increase the heating of the edges and improve the uniformity of heating to the $\pm 8 \%$ level. These calculations assumed an optically thin isotropic emitter; for the case of an optically thick Lambertian source, the viewfactor results change only slightly.

To improve the detected signal, one needs to increase the number of potential scattering electrons, the probe solid angle subtended at the scatterer, and collection solid angle. The first criterion is achieved by maximizing the x-ray mean free path (MFP) by using the lowest convenient atomic number $\mathrm{Z}$ material in solid form, Be. There is an additional advantage in using a low $\mathrm{Z}$ sample in that there is a reduction in the bound electron scattering contribution $\left(Z_{\mathrm{tb}}{ }^{2}+\mathrm{Z}_{\mathrm{wb}}\right)$ relative to the free electron contribution $\sim \mathrm{Z}_{\mathrm{f}}$. Hence, any undesirable coherent scattering due to the spectral wings of the probe by bound electrons can be kept negligible compared to the dispersed free electron scattering contribution. For solid density $\mathrm{Be}$ at $\approx 10 \mathrm{eV}$, calculations [17] suggest $Z_{\mathrm{tb}} \approx 1, Z_{\mathrm{wb}} \approx 2$ and $\mathrm{Z}_{\mathrm{f}} \approx 1$, yielding a ratio of bound electron to free electron scattering of only 3 to 1 . This reduces the spectral contrast required of both the probe source and detection system. It is also instructive to consider the relative importance of the inevitable and undesirable scattering from shields and target mounting stalks. The tightly bound electron scattering contribution / unit area will be $\sim \mathrm{Z}_{\mathrm{tb}}{ }^{2} \mathrm{MFP}$, hence $\sim \mathrm{Z}^{-1.5}$ since the MFP scales as $Z^{-3.5}$. For weakly bound electrons, the Compton scattering contribution will be $\sim Z_{\mathrm{wb}} \mathrm{MFP} \sim \mathrm{Z}^{-}$ 2.5. Hence, by constructing the shields of high $\mathrm{Z}$ material (e.g. Au) will minmize this problem.

A Be cylinder $\sim 0.5 \mathrm{~mm}$ in diameter by $0.5 \mathrm{~mm}$ in length $\mathrm{x}$ has been chosen as the optimum sample, heated by $4-5 \mathrm{keV}$ pump photons which have a $1 \mathrm{~mm}$ MFP in cold Be. A laser pulse length of $1 \mathrm{~ns}$ will extract maximum power $(20 \mathrm{TW})$ and energy $(\mathrm{E}=20 \mathrm{~kJ})$ from $42-45$ laser beams while restricting the fraction of sample compression and disassembly (disassembly rate $\approx$ $20 \mu \mathrm{m} / \mathrm{ns}$ at a few eV plasma temperature). By aperturing, as shown in Fig. 5a, one can also avoid a view of the shock compressed region which penetrates into the Be at a rate of 50-75 
$\mu \mathrm{m} / \mathrm{ns}$. A CsI layer, which has been shown to have $1.5 \%$ conversion efficiency to $4-5 \mathrm{keV} \mathrm{L-}$ shell $\mathrm{x}$-rays at a $2 \times 10^{15} \mathrm{~W} / \mathrm{cm}^{2}$ laser intensity, provides the pump source [67]. We calculate that the energy absorbed per $\mathrm{Be}$ atom is $20 \mathrm{eV}$, which should provide a few $\mathrm{eV}$ solid density plasma. Higher temperatures can be achieved by doping a smaller Be sample with a more absorptive element, at the expense of reducing the number of potential scatterers, or by using a softer pump photon energy with shorter MFP, at the expense of increased heating non-uniformity. We note that the separation into pump and probe beams allows for varying the sample temperature while keeping the number of probe photons fixed. The energy deposited in the Be sample could be measured on separate shots by using a calibrated crystal spectrometer viewing the transmitted $\mathrm{x}$ rays from planar CsI targets with and without Be overcoats. The combination of electron temperature, density, ionization state and energy absorbed would allow us to address the critical issue of how the energy is partitioned after equilibration has occurred in a low temperature dense plasmas.

To choose the scattering photon energy one requires that it is spectrally clear of the pump source spectrum, that it is high enough such that its MFP is at least twice the sample size, yet not so high that its production efficiency $\eta_{\mathrm{T}}$ suffers.[68]. A likely candidate is the He-like $\mathrm{V}$ resonance line at $5.2 \mathrm{keV}(\lambda=2.4 \AA)$, with a $1.4 \mathrm{~mm}$ MFP in Be.

The probe solid angle subtended at the sample has been maximized while still allowing for passage of the scattered photons, and shielding of the spectrometer from the probe source (see Fig. 4). In addition, the probe can be considered non-invasive relative to the pump source, as it has $<1 \%$ of the pump strength, having 0.33 the laser energy $\left(E_{T}=6 \mathrm{~kJ}\right), 0.2$ the hard $\mathrm{x}$-ray conversion efficiency and 0.1 the solid angle as seen by the Be sample. Potential heating by soft $\mathrm{x}$-rays from the probe source is eliminated by placing $50 \mu \mathrm{m}$ of Be between the source and the Be sample. 
A near backscattering geometry $\left(\theta=160^{\circ}\right)$ has been chosen for several reasons. First, blurring of the magnitude of the scattering vector $\mathbf{k}_{\mathbf{s}}$ due to a finite range of scattering angles $d \theta$ in the experiment can be minimized by operating close to the backscatter direction, since $\mathrm{k}_{\mathrm{s}} \approx$ $2 \mathrm{k} \sin (\theta / 2)$, and hence $\mathrm{dk}_{\mathrm{s}} / \mathrm{k}_{\mathrm{s}}=\mathrm{d} \theta /(2 \tan (\theta / 2))$ approaches 0 as $\theta$ approaches $180^{\circ}$. In the current experimental geometry $\theta$ and $\mathrm{d} \theta$ are set by the desire to minimize the probe stand-off distance and keep $\theta$ close to $180^{\circ}$, while simultaneously accommodating for realistic spot sizes and probe laser intensities $\left(\approx 10^{15} \mathrm{~W} / \mathrm{cm}^{2}\right)$. This has led to $\theta=160^{\circ}, \mathrm{d} \theta= \pm 15^{\circ}$, and hence an acceptable blurring level of $\mathrm{dk}_{\mathrm{s}} / \mathrm{k}_{\mathrm{s}}=2 \%$. Second, the scattering efficiency for unpolarized light is $2 \mathrm{x}$ greater near $180^{\circ}$ than it is for the more traditional $90^{\circ}$ geometry. Third, the magnitude of the scattering vector $\mathbf{k}_{\mathbf{s}}$ is maximized as $180^{\circ}$ is approached, allowing us to access the incoherent scattering regime $\left(\alpha=1 / \mathrm{k}_{\mathrm{s}} \lambda_{\mathrm{D}}<1\right)$ and/or shorter wavelength correlations without having to resort to even shorter wavelength probes or having to increase the Debye length by further heating the plasma. Fourth, the advantageous spectral separation of coherent and incoherent components due to Compton scattering of the photons from both weakly bound electrons and free electrons of mass $\mathrm{m}_{\mathrm{e}}$ is greatest at $\theta=180^{\circ}$, as given by $\mathrm{dh} v / \mathrm{h} v \approx-\left(\mathrm{hv} / \mathrm{m}_{\mathrm{e}} \mathrm{c}^{2}\right)(1-\cos \theta) \approx 0.02$ for $\mathrm{h} v \approx 5 \mathrm{keV}$.

The usual Thomson scattering electron feature will be centered around this downshifted incoherent Compton scattering peak. Note that the approximate Doppler broadened halfwidth of the backscattered electron feature for electrons of velocity $\mathrm{v}$ is $\approx 2(\sqrt{2}) \mathrm{v} / \mathrm{c}$ for scattering parameter $\alpha<1$ is less than the Compton downshift for plasma temperatures below $30 \mathrm{eV}$. The spectral blurring $\delta \mathrm{h} v / \mathrm{h} v$ due to the finite range of scattering angles $\mathrm{d} \theta$ for Compton scattering is also acceptably small, as given by $\delta \mathrm{h} v / \mathrm{h} v=\left(\mathrm{hv} / \mathrm{m}_{\mathrm{e}} \mathrm{c}^{2}\right) \sin \theta \mathrm{d} \theta, \approx 0.001$ for $\mathrm{h} v=5 \mathrm{keV}, \theta=160^{\circ}$ and $\mathrm{d} \theta= \pm 15^{\circ}$. 
In addition to the spectral isolation, we envisage delaying the probe by $1 \mathrm{~ns}$ and detecting the spectrally dispersed scattered photons by a standard microchannel-plate-based (MCP) framing camera to provide temporal isolation from the background of pump photons. The product of MCP efficiency and filter transmission required to protect the spectrometer and detector is estimated at $\eta_{d}=1 \%$. At the downshifted energy of $5.1 \mathrm{keV}$, the diffraction from the efficient Highly Oriented Pyrolytic Graphite (HOPG) 002 Bragg crystal planes [69] (2d spacing = $6.7 \AA$, integrated reflectivity $\mathrm{R}=2 \times 10^{-3}$ radians) is available at a moderately dispersive $21^{\circ} \mathrm{Bragg}$ angle. This level of high integrated reflectivity can be attained while simultaneously maintaining adequate spectral resolution $\Delta v / v=.003$ by using the natural Johann focussing [70,71] of a mosaic crystal [72] such as HOPG. The maximum reasonable collection angle in the nondispersive direction is set by the acceptable detector solid angle, $\Omega_{\mathrm{x}} \approx 0.1$ radians. The dispersion at the detector can be set such that the source broadening of $0.5 \mathrm{~mm}$ translates to a spectral broadening of only $\Delta v / v=0.003$, small compared to the minimum probe line width (0.002) and the widths of the Thomson scattered electron features (0.03) (see Fig. 6). A typical 3-cm-long MCP active region will hence accommodate a total spectral coverage $\mathrm{dv} / \mathrm{v}$ of 0.25 , allowing the full scattered spectrum to be detected on a single shot. In particular, the unbroadened, unshifted probe spectrum conveniently obtained from the nearby coherent scattering component can be used to deconvolve the total source plus instrument spectral response from the Thomson scattered spectrum.

The expected signal can now be estimated. The number of detected photons $\mathrm{N}$ in the electron feature is given by: $\mathrm{N}=\left(\mathrm{E}_{\mathrm{T}} \eta_{\mathrm{T}} / \mathrm{hv}\right)\left(\Omega_{\mathrm{T}} / 4 \pi\right)\left(\mathrm{n}_{\mathrm{e}} \sigma_{\mathrm{T}} \mathrm{x} /\left(\alpha^{2}+1\right)\right)\left(\Omega_{\mathrm{x}} \mathrm{R} / 4 \pi\right) \eta_{\mathrm{d}}$. Recalling that $\mathrm{E}_{\mathrm{T}}=5 \mathrm{~kJ}$, $\eta_{\mathrm{T}}=.003, \mathrm{~h} v=5 \mathrm{keV}, \Omega_{\mathrm{T}} / 4 \pi=0.01, \mathrm{n}_{\mathrm{e}} \sigma_{\mathrm{T}} \mathrm{x} /\left(\alpha^{2}+1\right)=0.002-0.003, \Omega_{\mathrm{x}} \mathrm{R} / 4 \pi=2.5 \times 10^{-5}$, and $\eta_{\mathrm{d}}=$ 0.01 leads to $\mathrm{N}=10,000$ - 15,000 detected photons in the Thomson scattered spectrum. Distributed over the 10 spectrally resolved bins, the expected signal-to-noise (SNR) ratio due to photon statistics is excellent, 30-40. It is instructive to consider how the signal scales with laser 
energy $\mathrm{E}$ and partitioning of pump and probe laser energy. For a given desired sample temperature, the number of heated sample atoms $\sim \mathrm{fE}$, where $\mathrm{f}$ is the fraction of laser energy used for the heater plasma. The number of probe photons reaching a given sample atom in a scaled experiment is then $\sim(1-\mathrm{f}) \mathrm{E}$. The signal for fixed detector solid angle varies as the product of the number of potential scatterers and number of probe photons reaching a given atom, $\sim(1-\mathrm{f}) \mathrm{fE}^{2}$. This yields a broad optimum around $\mathrm{f}=0.5$, with only a factor of $30 \%$ drop in signal for the current $f \approx 0.8$ design point. More interesting is the strong $E^{2}$ scaling, which explains why smaller laser facilities with 1-10\% of the energy would be insufficient.

\section{Future Applications}

We have shown that $\mathrm{x}$-ray Thomson scattering is most easily applied to low $\mathrm{Z}$ plasmas by virtue of their long MFPs. These are also the plasmas that are most difficult to observe by spectroscopic means since they have no bound transitions in the hard $\mathrm{x}$-ray regime. A future $\mathrm{x}$ ray Thomson scattering application therefore includes measuring the adiabat (essentially the ratio $\mathrm{T}_{\mathrm{e}} / \mathrm{T}_{\mathrm{F}}$ ) and density of deuterium and tritium ICF fuel, both driven in planar and spherically convergent geometry. The $T_{e}-n_{e}$ regime spanned by imploding fuel in igniting ICF capsules is shown in Fig. 7. Peak fuel electron densities of $10^{26} \mathrm{~cm}^{-3}$ with $\mathrm{Te} \leq \mathrm{T}_{\mathrm{F}} \approx 1 \mathrm{keV}$ are required for efficient assembly and subsequent burn of the fuel after ignition [8]. The fraction scattered can be of order $10 \%$ for such fuel areal densities of $10^{23} \mathrm{~cm}^{-2}$. The expected HWHM of the x-ray Thomson scattering spectrum at such high values of $T_{e}$ and $T_{F}$ is of order 0.1 , easily resolvable.

Another area where $x$-ray Thomson scattering could provide a non-invasive $T_{e}$ measurement is for supersonic radiation transport studies.[73-75]. Typical electron densities and temperatures range from $10^{21}$ to $10^{23} \mathrm{~cm}^{-3}$ and from 50 to $200 \mathrm{eV}$ (see Fig. 8), corresponding to foam samples heated in a hohlraum environment. One could switch to $90^{\circ}$. scattering, and by using a 
cylindrical Von Hamos geometry Bragg crystal [76], acquire space-resolved data to provide an axial profile of the temperature. The temperature could either be extracted using the full spectrum recorded in gated mode or extracted from streaked records of the emissivity at two frequency detunings (“2-color spectroscopy”).

Finally, we expect that future picosecond to sub-picosecond x-ray laser sources (e.g. the x-ray XFEL proposal for the Stanford Linear Accelerator, and at TESLA DESY Hamburg) could be used as $\mathrm{x}$-ray Thomson scattering sources. The advantage here is that the short pulse duration allows for pumping and probing much smaller samples ( $\mu \mathrm{m}$-scale vs mm-scale) since the time allowed for disassembly is $1000 \mathrm{x}$ shorter. Coupled with the collimation provided by an x-ray laser, the requirements on pump and probe X-ray energy are of order $10^{6}$ less ( $\mathrm{mJ}$ instead of $\left.\mathrm{kJ}\right)$.

\section{Summary}

High frequency X-ray Thomson scattering has been shown to be a viable technique for inferring microscopic parameters of dense plasmas opaque to optical probing. We have developed an $\mathrm{x}$ ray Thomson scattering experiment fully optimized for the Omega laser facility capabilities which should provide high quality spectral data on a single shot. We expect a successful attempt at extending the full versatility of laser Thomson scattering to the x-ray regime to open the door for detailed dense plasma studies of great interest to the high energy density and plasma physics communities.

\section{Acknowledgments}

Work performed under the auspices of the U.S. Department of Energy by the University of California Lawrence Livermore National Laboratory under contract number W-7405-ENG-48. 


\section{References}

[1] Hansen JP and McDonald IR. Thermal relaxation in a strongly coupled two-temperature plasma. Phys. Lett. A 1983;97A:42-44.

[2] Boercker DB, Lee RW, and Rogers FJ. Strong coupling effects on plasma line shapes and Thomson scattering signals. J. of Phys. B 1983;16:3279-3290.

[3] Ichimaru S and Tanaka S. Theory of interparticle correlations in dense, high-temperature plasmas. V. Electric and thermal conductivities. Phys. Rev. A 1985;32:1790-1798.

[4] Boercker DB and More RM. Statistical mechanics of a two-temperature, classical plasma. Phys. Rev. A 1986;33:1859-1869.

[5] Forsman A, Ng S, Chiu G et al. Interaction of femtosecond laser pulses with ultrathin foils, Phys. Rev. E 1998;58:R1248-1251.

[6] Price D, More RM, Walling RS et al. Absorption of ultrashort laser pulses by solid targets heated rapidly to temperatures 1-1000 eV. Phys. Rev. Lett. 1995;75:252-255.

[7] Setsuhara Y, Azechi H, Miyanaga N et al. Secondary nuclear fusion reactions as evidence of electron degeneracy in highly compressed fusion fuel. Laser Part. Beams 1990:8:609620.

[8] Lindl J. Development of the indirect-drive approach to inertial confinement fusion and the target physics basis for ignition and gain. Phys. Plasmas 1995;2:3933-4024.

[9] Peyrusse O, Busquet M, Kieffer JC et al. Generation of hot solid-density plasmas by laser radiation pressure confinement. Phys. Rev. Lett. 1995;75:3862-3865.

[10] Woolsey NC, Asfaw A, Hammel KB et al. Spectroscopy of compressed high energy density matter. Phys. Rev. E 1996;53:6396-6402.

[11] Meyer-ter-Vehn J, Oparin A, and Aoki T. Options for laser compression of matter to study dense-plasma phases at low entropy, including metallization of hydrogen. In: Miley G, 
Nakai S, editors. 12th International Conference on Laser Interaction and Related Plasma Phenomena, vol. 369. Woodbury NY: AIP, 1996. p. 347-356.

[12] Nantel M, Ma G, Gu S et al. Pressure ionization and line merging in strongly coupled plasmas produced by 100-fs laser pulses. Phys. Rev. Lett. 1998;80:4442-4445.

[13] Theobald W, Hassner R, Kingham R et al. Electron densities, temperatures, and the dielectric function of femtosecond-laser-produced plasmas. Phys. Rev. E 1999;59:35443553.

[14] Ichimaru. S. Strongly coupled plasmas: High density classical plasmas and degenerate electron liquids. Rev. Mod. Phys 1982;54:1017-1059.

[15] van Horn HM. The Equation of State in Astrophysics. Cambridge:Cambridge University Press, 1994..

[16] Rose SJ. The effect of degeneracy on the scattering contribution to the radiative opacity. Astrophys. J. Lett. 1995;453:L45-L47.

[17] Peyrusse O. A method for calculating the effect of ionization on Thomson scattering. J. Quant. Spectrosc. Radiat. Trans. 1990;43:397-405.

[18] Linnebur EJ and Duderstadt JJ. Theory of light scattering from dense plasmas. Phys. Fluids $1973 ; 16: 665-674$.

[19] Theimer O and Behl YK. Electron density fluctuations in a plasma with collision frequency proportional to speed. Plasma Phys. 1977;19:1119-1128.

[20] Cauble R and Boercker DB. Dynamic structure factors in two-component plasmas. Phys. Rev. A 1983;28:944-951.

[21] DeSilva AW and Kunze HJ. Experimental study of the electrical conductivity of strongly coupled copper plasmas. Phys. Rev. E 1994;49:4448-4454.

[22] Krisch I and Kunze HJ. Measurements of electrical conductivity and the mean ionization state of nonideal aluminum plasmas. Phys. Rev. E 1998;58:6557-6564. 
[23] DeSilva AW and Katsouros JD. Electrical conductivity of dense copper and aluminum plasmas. Phys. Rev. E 1998;57:5945-5951.

[24] Benage JF, Jr., Shanahan WR, Sherwood EG et al. Measurement of the electrical resistivity of a dense strongly coupled plasma. Phys. Rev. E 1994;49:4391-4396.

[25] Rozmus W and Offenberger AA. Thermal conductivity for dense, laser-compressed plasmas. Phys. Rev. A 1985;31:1177-1179.

[26] $\mathrm{Ng} \mathrm{A}$, Forsman A, and Chiu G. Electron thermal conduction waves in a two-temperature, dense plasma, Phys. Rev. Lett. 1998;81:2914-2917.

[27] Mostovych AN, Kearney KJ, Stamper JA et al. Measurements of plasma opacity from laser-produced optically thin strongly coupled plasmas. Phys. Rev. Lett. 1991;66:612-615.

[28] Eidmann K, Bar-Shalom A, Saemann A et al. Measurement of the extreme UV opacity of a hot dense gold plasma. Europhys. Lett. 1998;44:459-464.

[29] Gauthier P, Rose SJ, Sauvan P et al. Modeling the radiative properties of dense plasmas, Phys. Rev. E 1998;58:942-50.

[30] Davidson SJ, Nazir K, Rose SJ et al. Short-pulse laser opacity measurements. J. Quant. Spectrosc. Radiat. Trans. 2000;65:151-160 .

[31] Liberman D and Albritton J. Dense plasma equation of state model, J. Quant. Spectrosc. Radiat. Trans. 1994;51:197-200.

[32] Perrot F and Dharma-Wardana MWC. Equation of state and transport properties of an interacting multispecies plasma: application to a multiply ionized Al plasma. Phys. Rev. E $1995 ; 52: 5352-5367$.

[33] Landen OL and Winfield RJ. Laser scattering from dense cesium plasmas. Phys. Rev. Lett. $1985 ; 54: 1660-1663$.

[34] Bennett GR, Wark JS, Heading DJ et al. Production of strongly coupled plasmas by the laser irradiation of thin metallic films confined within micrometer-scale gaps by transparent insulators. Phys. Rev. E 1994;50:3935-3942. 
[35] Mostovych AN and Chan Yung. Reflective probing of the electrical conductivity of hot aluminum in the solid, liquid, and plasma phases. Phys. Rev. Lett. 1997;79:5094-5097.

[36] Milchberg HM, Freeman RR, and Davey SC. Resistivity of a simple metal from room temperature to 10/sup 6/ K. Phys. Rev. Lett. 1988;61:2364-2367.

[37] Ng A, Celliers P, Forsman A et al. Reflectivity of intense femtosecond laser pulses from a simple metal. Phys. Rev. Lett. 1994;72:3351-3354.

[38] Mahdieh MH and Hall TA. Optical reflectivity of dense plasmas produced by laser driven shock waves. J. of Phys. D 1997;30:588-592.

[39] Quoix C, Hamoniaux G, Antonetti A et al. Ultrafast plasma studies by phase and amplitude measurements with femtosecond spectral interferometry. J. Quant. Spectrosc. Radiat. Trans. 2000;65:455-462.

[40] Landen OL, Stearns DG, and Campbell EM. Measurement of the expansion of picosecond laser-produced plasmas using resonance absorption profile spectroscopy. Phys. Rev. Lett. $1989 ; 63: 1475-1478$.

[41] Landen OL and Alley WE. Dynamics of picosecond-laser-pulse plasmas determined from the spectral shifts of reflected probe pulses. Phys. Rev. A 1992;46:5089-5100.

[42] Bradley DK, Kilkenny J, Rose SJ et al. Time-resolved continuum-edge-shift measurements in laser-shocked solids. Phys. Rev. Lett. 1987;59:2995-2998.

[43] Hall TA, Djaoui A, Eason RW et al. Experimental observation of ion correlation in a dense laser-produced plasma. Phys. Rev. Lett. 1988;60:2034-2037.

[44] Riley D, Willi O, Rose SJ et al. Blue shift of the K absorption edge in laser-shocked solids. Europhys. Lett. 1989;10:135-140.

[45] Heading DJ, Bennett GR, Wark JS et al. Novel plasma source for dense plasma effects. Phys. Rev. Lett. 1995;74:3616-3619.

[46] Shiwai BA, Djaoui A, Hall TA et al. Improvements to ion-correlation experiments in dense plasmas. Laser Part. Beams 1992;10:41-51. 
[47] Oks EA, Boddeker S, and Kunze HJ. Spectroscopy of atomic hydrogen in dense plasmas in the presence of dynamic fields: Intra-Stark spectroscopy. Physical Review A $1991 ; 44: 8338-8347$.

[48] Nardi E. Scattering of x-rays by the bound and free electrons in dense plasmas. Phys. Rev. A 1991;43:1977-1982.

[49] Woolsey NC, Riley D, and Nardi E. Kilovolt x-ray scattering from a plasma. Rev. Sci. Instrum. 1998;69:418-424.

[50] Nardi E, Zinamon Z, Riley D et al. X-ray scattering as a dense plasma diagnostic. Phys. Rev. E 1998;57:4693-4697.

[51] Riley D, Woolsey NC, McSherry D et al. X-ray scattering from a radiatively heated plasma. J. Quant. Spectrosc. Radiat. Trans. 2000;65:463-470.

[52] Evans DE and Katzenstein J. Laser light scattering in laboratory plasmas. Rep. Prog. Phys. $1969 ; 32: 207-271$.

[53] Cameron SM, Tracy MD, Estabrook KG et al. Two-dimensional electron density, temperature, and radial drift profiles of a laser plasma by $266 \mathrm{~nm}$ collective Thomson scattering. Rev. Sci. Instrum. 1992;63:5259-5265 .

[54] Glenzer SH., Rozmus W, MacGowan BJ et al. Thomson scattering from high-Z laserproduced plasmas. Phys. Rev. Lett. 1999;82:97-100.

[55] Montgomery DS, Johnson RP, Cobble JA et al. Characterization of plasma and laser conditions for single hot spot experiments. Laser Part. Beams 1999;17:349-359.

[56] McNelis NB and DeSilva AW. Experimental tests of light scattering theory in plasmas. Plasma Phys. 1982;24:1261-75.

[57] Liboff RL. Criteria for physical domains in laboratory and solid-state plasmas. J. Appl. Phys. 1984;56:2530-2535.

[58] Baldis HA, Dunn J, Foord ME et.al. X-ray Thomson scattering: towrads diagnostics for solid density plasmas. To appear in Proc. 7th Intl. Conf. on X-ray Lasers 2001. 
[59] Hubbell JH, Weigele WJ, Briggs EA et al. Atomic form factors, incoherent scattering functions, and photon scattering cross sections. J. Phys. Chem. Ref. Data 1975;4:471-533.

[60] Ross PA and Kirkpatrick P. Effect of electron binding upon the magnitude of the Compton shift. Phys. Rev. 1934;46:668-673.

[61] Bloch F. Contribution to the theory of the Compton-line. Phys. Rev. 1934;46:674-687.

[62] Weigele WJ, Tracy PT, and Henry EM. Compton effect and electron binding. Am. J. Phys. 1966;34:1116-1121.

[63] Mandl F. Statistical Physics. New York:Wiley, 1971. p. 290.

[64] Chandrasekhar S. The Compton scattering and the new statistics. Proc. Roy. Soc. 1929;A125:231-237.

[65] Liberman DA. Self-consistent field model for condensed matter. Phys. Rev. B 1979;20:4981-4989.

[66] Soures JM, McCrory RL, Verdon CP, et. al. Direct-drive laser fusion experiments with the Omega 60-beam, > 40 kJ, ultraviolet laser system. Phys. Plasmas 1996;3: 2108-2112.

[67] Back CA, Grun J, Decker C, Suter LJ et al. Efficient multi-keV underdense laser-produced plasma radiators. Submitted to Phys. Rev. Lett. 2000.

[68] Glendinning SG, Amendt P, Budil KS et al. Laser plasma diagnostics of dense plasmas. In: Kyrala G, editor. Applications of Laser Plasma Radiation II, vol. 2549. Bellingham: SPIE,.1995. p.29-39.

[69] Kestenbaum HL. X-ray diffraction from graphite in the energy range 2 to $8 \mathrm{keV}$. Appl. Spectrosc. 1973;27:454-456.

[70] Sanchez del Rio M, Bernstorff S, Savoia A, Cerrina F. A conceptual model for ray tracing calculations with mosaic crystals. Rev. Sci. Instrum. 1999;63:932-935.

[71] Mizogawa T. An x-ray spectrometer using a 2D PSPC and a flat crystal. Phys. Scripta 1977;T73:403-404. 
[72] Ohler M, Baruchel J, Moore AW, et. al. Direct observation of mosaic blocks in highly oriented pyrolytic graphite. Nuc. Instrum. Meth. 1997;129:257-260.

[73] Back CA, Bauer JD, Landen OL, et. al. Detailed measurements of a diffusive supersonic wave in a radiatively heated foam. Phys. Rev. Lett. 2000;84:274-277.

[74] Back CA, Bauer JD, Hammer JH, et. al. Diffusive supersonic x-ray transport in radiatively heated foam cylinders. Phys. Plasmas 2000;7:2126-2134.

[75] Edwards J, Glendinning SG, Suter LJ, et. al. Turbulent gydrodynamics experiment using a new plasma piston. Phys. Plasmas 2000;7:2099-2107.

[76] Yaakobi B, Turner RE, Schnopper HW et al. Focusing X-ray spectrograph for laser fusion experiments. Rev. Sci. Instrum. 1979;50:1609-1611. 


\section{Figure Captions}

Figure 1 Electron density and temperature parameter space showing Fermi degenerate (upper left shaded) and strongly coupled plasma regimes (middle shaded), where lower regime edges are set by $\mathrm{T}_{\mathrm{e}}=\mathrm{T}_{\mathrm{F}}$ and $\Gamma_{\mathrm{ee}}=1$, respectively. The dashed line at $\approx 2 \times 10^{23} \mathrm{~cm}^{-3}$ represents the solid Be equilibrium electron density (conduction and free electrons) [17]. Typical regions for Thomson backscattering, i.e., where $\theta=180^{\circ}$, are denoted by solid lines that bound $\alpha=0.1$ and 0.3 for probe wavelengths $=2400$ and $2.4 \AA$.

Figure 2 Schematic of spectrally-resolved x-ray backscattering spectrum expected, with information provided by each feature noted as $\mathrm{f}(\mathrm{)}$. The shape of the electron Thomson scattered feature will change from a parabolic Fermi degenerate distribution for $\mathrm{T}_{\mathrm{e}}<<\mathrm{T}_{\mathrm{F}}$ to a Gaussian Boltzmann distribution for $\mathrm{T}_{\mathrm{e}} \gg \mathrm{T}_{\mathrm{F}}$. The peak labeled Compton scattering will be a mixture of scattering from weakly bound electrons and low frequency ion acoustic-driven Thomson scattering from free electrons for values of $\alpha>0.1$.

Figure 3 Calculated Thomson backscattered spectra for various ratios of $\mathrm{T}_{\mathrm{e}} / \mathrm{T}_{\mathrm{F}}$ for $\mathrm{T}_{\mathrm{F}}=15 \mathrm{eV}$. Solid, long dashed and short dashed correspond to $\mathrm{T}_{\mathrm{e}} / \mathrm{T}_{\mathrm{F}}=0.1,0.2$ and 0.4 . The spectral shift corresponding to an electron velocity component equal to the Fermi velocity is denoted by a vertical line. Note that only one side of spectrum shown, and that $\Delta v=0$ corresponds to the Compton shifted frequency.

Figure 4 Schematic, drawn to scale, of proposed experimental set-up at Omega. Target is cylindrically symmetric about collection axis (dashed line). $20 \mathrm{~kJ}$ irradiates a thin CsI-coated plastic foil surrounding the central cylindrical Be sample, producing the 4-5 keV x-ray heating 
source. A $5 \mathrm{keV}$ Thomson scattering probe is produced on the left by irradiating a thin vanadium annular foil with 5-6 kJ of laser energy.

Figure 5 a) Cylindrical target geometry with overhang optimized to minimize gradients in $\mathrm{Be}$. b) Calculated distribution function of energy absorbed per atom throughout Be sample visible to spectrometer. Solid and dashed lines correspond to cases with (outer/inner length $=1.5$ ) and without overhang geometry. Case plotted is for Be cylinder inner length, outer length and radius $=0.5,0.75$ and 0.25 of pump photon mean free path.

Figure 6 Spectra from probe source (solid line), convolved with source size (dashed line), further convolved with spectrometer resolution (dotted line), compared with expected unconvolved scattered spectrum $\left(\mathrm{T}_{\mathrm{e}}=3 \mathrm{eV}, \mathrm{T}_{\mathrm{F}}=15 \mathrm{eV}\right)$ (dashed-dotted line).

Figure 7 Plasma parameter space for ICF DT fuel regime during compression, showing $\alpha=0.1$ and 1 curves for $\lambda=2.4 \AA, \theta \approx 180^{\circ}$. Dashed line denotes separation between upper Fermi degenerate and lower non-degenerate plasma regimes.

Figure 8 Plasma parameter space for radiatively heated foam regime, showing $\alpha=0.1$ and 0.03 curves for $\lambda=2.4 \AA, \theta \approx 180^{\circ}$. 


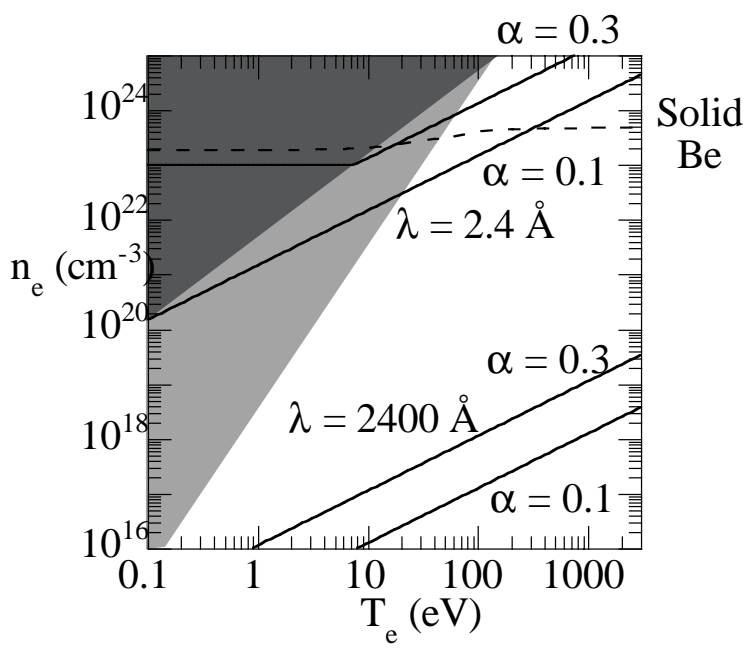

Figure 1 


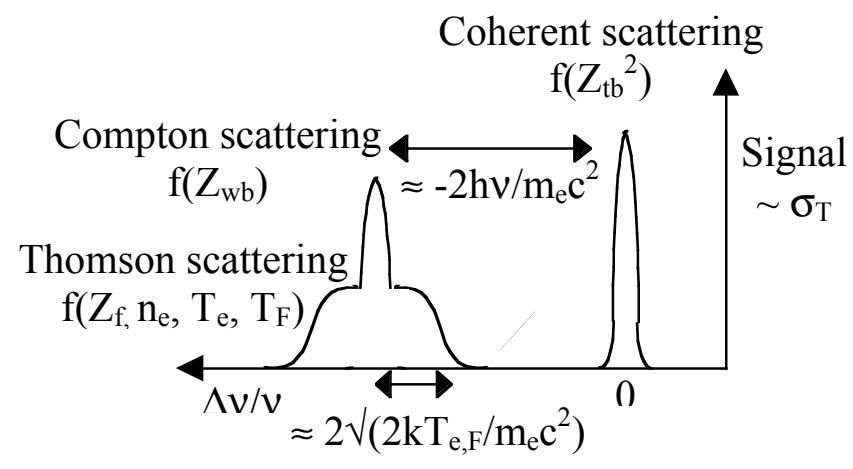

Figure 2 


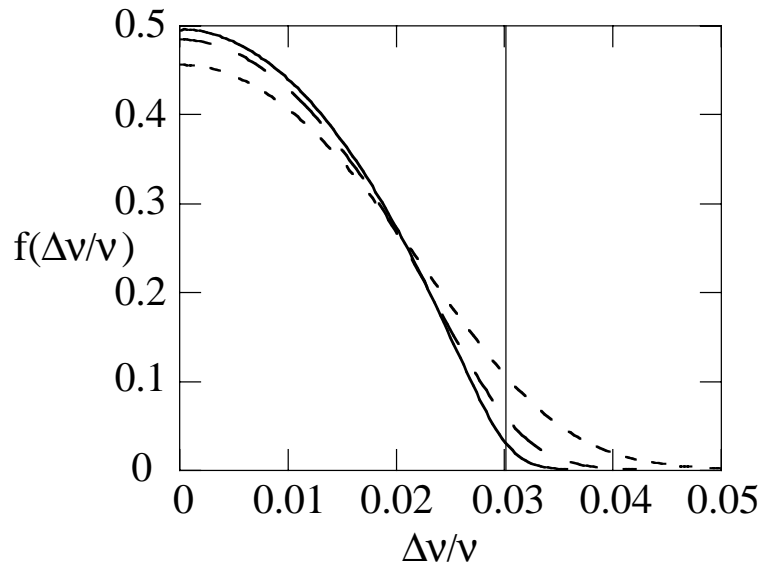

Figure 3 


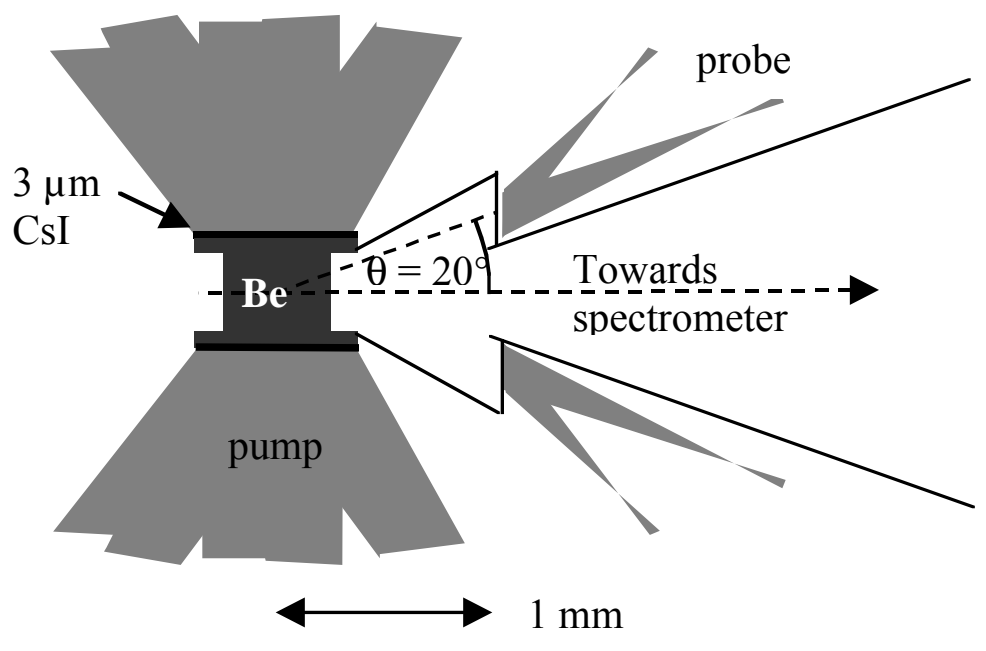

Figure 4 
a)

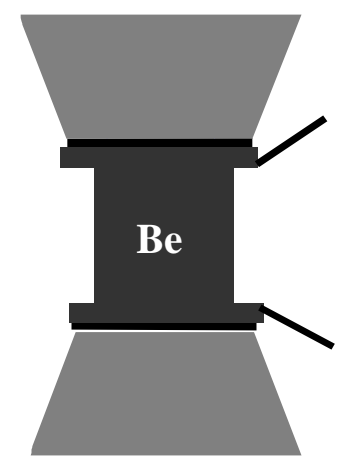

b)

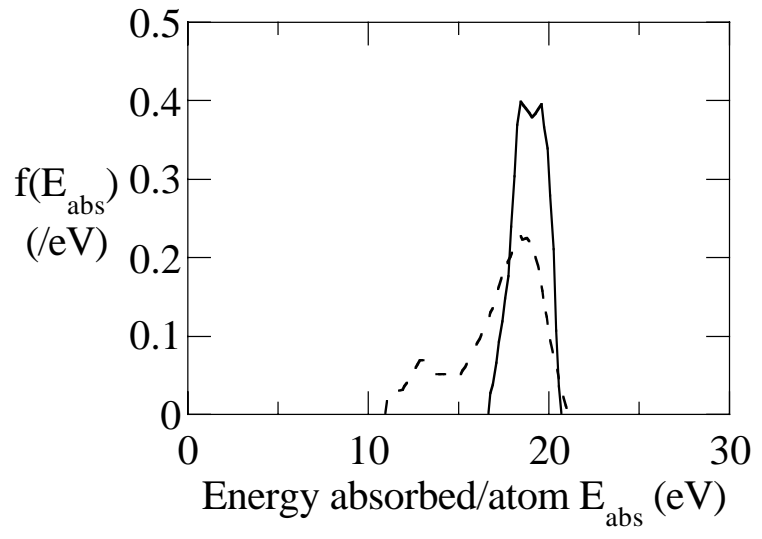

Figure 5 


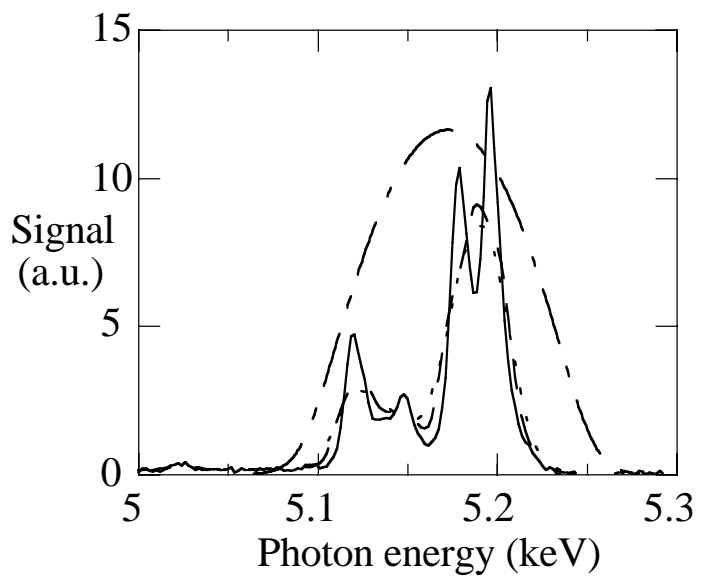

Figure 6 


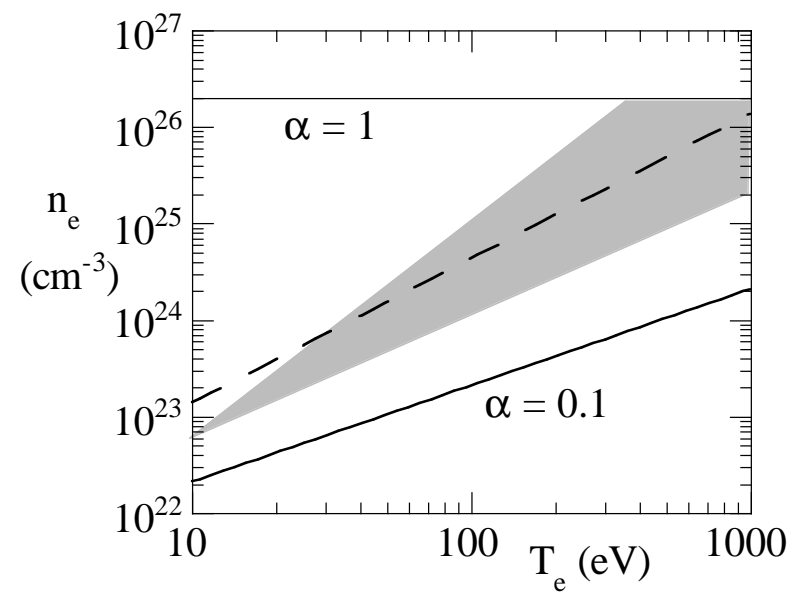

Figure 7 


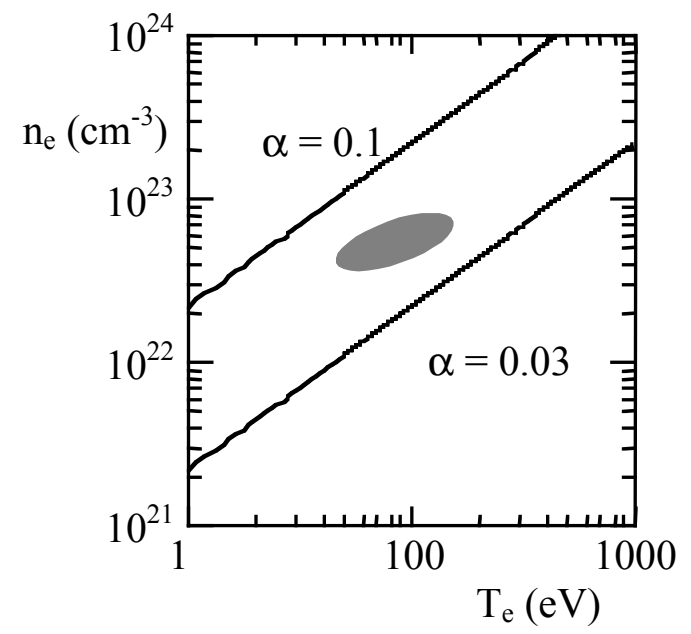

Figure 8 